\author{
Military Technical College \\ Kobry El-Kobbah, \\ Cairo, Egypt
}

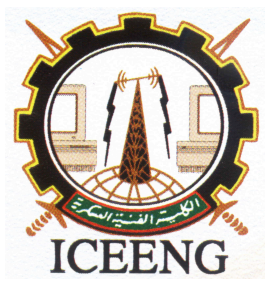

\author{
$11^{\text {th }}$ International Conference \\ on Electrical Engineering \\ ICEENG 2018
}

\title{
INTEGRITY PERFORMANCE OF EGYPTIAN ELORAN SYSTEM FOR MARITIME APPLICATION
}

\author{
Farag M. Bahr*, Ezz Eldin Abdelkawy ${ }^{\dagger}$, Samy A.Shedied ${ }^{\dagger}$
}

\section{Abstract}

For maritime applications, eLoran is considered as the most promising backup for Global Navigation Satellite System in case the use of navigation satellite signals is denied. We present a proposal study to establish eLoran navigation system for Egyptian maritime harbors to act as a national and international backup to GPS for maritime navigation, and other user sectors using MATLAB. The purpose of the Egyptian eLoran system is providing performance requirements for maritime Harbor Entrance Approach (HEA) on all Egyptian harbors. Accuracy, integrity, continuity and availability are the most interested requirements for HEA. The required levels of Integrity as a requirement of the International Maritime Organization had been verified over all the Egyptian harbors.

\section{Keywords:}

eLoran, Integrity, and Horizontal protection level

${ }^{*},+$ Egyptian Armed Forces. 


\section{INTRODUCTION}

The Global Positioning System (GPS) is most often the primary source of Position, Navigation and Timing (PNT) information. The recent concerns about vulnerability of GPS have sparked a renewed interest in the Loran PNT system. Enhanced Loran "eLoran" is the latest version of Loran PNT for longstanding and proven series of low frequency navigation systems which are used to provide back-up capabilities to GPS in maritime navigation [1]. eLoran is a terrestrial low-frequency system organized as chains each consisting of a master station with two or more secondary stations. eLoran meets international performance standards that allow it to serve as a backup to GPS in a great number of applications across multiple sectors [2]. The primary requirements of concern for Non Precision Approach (NPA) or Harbor Entrance Approach (HEA) are the accuracy, integrity, availability and continuity as depicted in Table 1. The user's receiver estimates the maximum likely error on the position-fix by calculating the Horizontal Protection Level (HPL), which is the basic method of providing positioning Integrity. If this error is below a specified Horizontal Alert Limit (HAL) then the fix is validated as 'good'. If the HPL breaches the HAL, then the fix is designated 'bad'.

\section{LEAST-SQUARES POSITIONING}

As eLoran is a ranging system, the pseudorange $\rho$ from the receiver to every eLoran transmitter can be represented as:

$$
\rho=R+P F+S F+A S F+\delta+\varepsilon+b
$$

Where:

$R$ is the true range

$S F$ is the Secondary Factor

$A S F$ is Additional Secondary Factor

$\delta \quad$ is variation in $\mathrm{PF}, \mathrm{SF}$ andASF
$P F$ is Primary Factor

$b$ is the receiver clock bias

$\varepsilon$ is remaining measurement errors

In this paper, we have assumed that eLoran position-fixing is derived using an over-determined Least-Squares solution of pseudorange observations. Since ground wave geodetic range measurements are not linearly related to position, we linearize the pseudorange equation, at a nominal position ( $x_{o}$, $\left.y_{o}\right)$ to give the linear matrix equation given by [3]:

$$
\Delta \rho=G . \Delta p
$$

Where Gis the direction cosine matrix or the geometry matrix and the correction vector $\Delta p$ is then used to update the position solution estimate. $\Delta \rho$ is the vector of corresponding changes in the measured pseudorange relative to its nominal value $\rho\left(x_{o}, y_{o}\right)$.

The equation (2) can be expanded to:

$$
\Delta \rho=\left[\begin{array}{ccc}
\sin \left(\beta_{1}\right) & \cos \left(\beta_{1}\right) & 1 \\
\sin \left(\beta_{2}\right) & \cos \left(\beta_{2}\right) & 1 \\
& \\
\sin \left(\beta_{n}\right) & \cos \left(\beta_{n}\right) & 1
\end{array}\right]\left[\begin{array}{c}
\Delta x \\
\Delta y \\
\Delta b
\end{array}\right]
$$

where $\beta_{n}$ are bearings of the stations from the receiver. 
The differences $\Delta x, \Delta y$ and $\Delta b$ represent corrections (expressed in meters) to be applied to the current position and receiver clock bias estimates. If the system contains more than three stations, we can get the solution by using equation given by:

$$
\Delta p=\left(G^{T} G\right)^{-1} G^{T} \Delta \rho
$$

If the measurement errors are uncorrelated but have different variance, we use Weighted Least Squares (WLS) position determination algorithm (which is usually the case in practice), the optimum solution is given by

$$
\Delta p=\left(G^{T}(\operatorname{var}(\Delta \rho))^{-1} G\right)^{-1} G^{T}(\operatorname{var}(\Delta \rho))^{-1} \Delta \rho
$$

Where var $(\Delta \rho)$ is Pseudorange measurement error and it is composed of variance of Time of Arrival (ToA) and variance of ASF map and differential Loran correction. Each term will have to be measured or estimated, and provided a-priori to the receiver so that each source of error can be accounted for.

\section{INTEGRITY CALCULATION}

Integrity is the ability of the system to inform a user when a signal or solution should not be used. For Loran, under nominal conditions, this is achieved by providing a horizontal protection level (HPL) that bounds the horizontal position error (HPE) [4]. Provision of an HPL for position-fixing is then found by integrating a position bound up to the five-nines confidence level. For the purposes of this work, we have used a Distance Root-Mean-Square (DRMS) -based HPL equation. The variance matrix of the position error can be obtained

$$
\begin{aligned}
& \operatorname{var}(\Delta p)=\left(\begin{array}{ccc}
\sigma_{x}^{2} & \sigma_{x y} & \sigma_{x b} \\
\sigma_{x y} & \sigma_{y}^{2} & \sigma_{y b} \\
\sigma_{x b} & \sigma_{y b} & \sigma_{b}^{2}
\end{array}\right)=\left(G^{T} \operatorname{var}(\Delta \rho) G\right)^{-1} \\
& \text { The DRMS accuracy can then be expressed simply as: } \\
& \alpha_{D R M S}=\sqrt{C(1,1)+C(2,2)} \\
& H P L=K_{H E A} \sqrt{C(1,1)+C(2,2)}
\end{aligned}
$$

\section{FACTORS AFFECTING DRMS ACCURACY OF POSITION}

A. Geometry of the Transmitter Stations in View

The locations of transmitter stations are generally selected to give better geometric configuration [3]. The effect of transmitter geometry on the accuracy is usually described by Horizontal Dilution of Precision (HDOP) factor. Assuming the pseudorange measurement errors are mutually uncorrelated and have equal variance $\sigma_{\rho}^{2}$.

$$
\operatorname{var}[\Delta \rho]=\sigma_{\rho}^{2} I
$$

Then, the variance matrix of position error can be reduced to 


$$
\operatorname{var}[\Delta p]=\left(\begin{array}{ccc}
\sigma_{x}^{2} & \sigma_{x y} & \sigma_{x b} \\
\sigma_{x y} & \sigma_{y}^{2} & \sigma_{y b} \\
\sigma_{x b} & \sigma_{y b} & \sigma_{b}^{2}
\end{array}\right)=\sigma_{\rho}^{2}\left(G^{T} G\right)^{-1}
$$

where $H$ part is dimensionless and depends only on the transmitter geometry. Hence, we can extract the HDOP from the following equation:

$$
\alpha_{D R M S}=\sqrt{\sigma_{x}^{2}+\sigma_{y}^{2}}=\sigma_{\rho} \sqrt{H_{1,1}+H_{2,2}}
$$

The locations of these new transmitters in Table 22are selected based on the coverage to give better HDOP over all harbors as shown inFig. 1.

\section{B. Accuracy of Signal Time-of-Arrival (ToA) Measurements}

Signal to noise ratio (SNR) of the received eLoran signal strongly determines the accuracy of ToA measurement. As eLoran signal is in the ground wave band, many error factors are compromising the noise and interference effects. These factors include the atmospheric noise, the cross-rate interference (CRI), sky wave interference, continuous-wave interference (CWI), and transmitter timing jitter and transmitting antenna impedance variations [3]. Sky wave interference, CRI and CWI are effectively mitigated by receiver processing algorithms but the residual errors from these sources may still be present in certain conditions. The calculation of SNR requires knowledge of the signal strength of the eLoran signal and the level of external noise at the same location. The main driver Pseudorange of variance is the signal-to-noise ratio (SNR) of the received signal because low SNR lead to high Pseudorange variance and given by:

$$
\operatorname{var}[\Delta \rho]=\sigma_{\rho}^{2}=\frac{337.5^{2}}{N . S N R}+\frac{c_{1}}{N}+c_{2}
$$

accounts for transmitter related noise, which is assumed to be $c_{1}=36 \mathrm{~m}^{2}$, accounts for other sources of variation in the pseudorange measurements $c_{2}=12 \mathrm{~m}^{2}, \mathrm{Nis}$ the number of pulses used in signal integration. Its value depend on integration time and time of Group Repetition Interval as depicted in the following equation

$$
N=\frac{8 T_{i}}{T_{G R I}}
$$

Ground wave field strength is calculated using propagation curves for different ground conductivities at $100 \mathrm{kHz}$ as shown in previous section. In this study, ground wave field strengths will be modeled using the Millington 'e s method as described in the ITU-R Recommendation [5].The signal strength of Loran station is shown in Fig. 2. The dominant noise source in the Loran band is atmospheric noise. Atmospheric noise is computed at different percentiles and generated based on the model presented in ITU Recommendation P372-9[6]. Fig. 3shows the distribution of the predicted average daytime noise over Egypt.Fig. 4 shows the SNR of eLoran station. An accurate estimate of pseudorange measurement variance under the assumption of white noise occur if the range of SNR 
from $-10 \mathrm{~dB}$ to $+40 \mathrm{~dB}$. In order to determine the number of pulses used in signal integration, we need to specify the value of time of Group Repetition Interval. The overall procedure of GRI selection is summarized in Fig .5.

\section{Accuracy of the ToA to Range Conversion}

The ground wave accumulates a delay compared to the expected propagation time over the same distance in free space. This delay is the accumulation of three components or factors and need to be accounted for when using measured pseudo-ranges to fix a position.

i. Primary Factor: Due to the refractive index of the atmosphere $\left.\left(\mathrm{n}_{P F}=1.000338\right)\right)$, the PF speed of the Loran signal through the atmosphere is given by:

$$
\mathrm{c}_{P F}=299,691,162 \mathrm{~m} / \mathrm{s}
$$

ii. Secondary Factor: Due to the electrical conductivity and curvature of the surface of the Earth an additional delay is incurred. Seawater is the best conducting surface, and it is possible to model the SF contribution from seawater using the equations described by P. Brunavs [8], as shown in Fig. 6.

iii. Additional Secondary Factor:Any land encountered with a surface conductivity lower than seawater will delay the signal even further. This additional delay is termed the Additional Secondary Factor or ASF. Work has shown it is not viable to determine ASF by modeling with sufficient accuracy for navigation $[8,9]$.

The current method for providing ASF data involves a comprehensive measurement campaign of an area, with the surveyed data then uploaded to the user's receiver. This survey will have been carried out on a particular day to fix the ASF values once and for all as shown in Fig. 7.Since ASF depends on the ground conductivity along the propagation path, any changes in conductivity will change the ASF.Over time there will be some variation from the fixed ASF values stored in the receiver due to Seasonal. It is known as temporal variations. Its effect appears on loran position accuracy in terms $\delta_{\text {temp }}^{2}, \sigma_{\text {temp }}^{2}$ which represent bias and variance in temporal variation respectively. So we can represent Pseudorange measurement error in meters.

$$
\operatorname{Var}[\Delta \rho]=\sigma_{\rho}^{2}=\frac{337.5^{2}}{N . S N R}+\frac{c_{1}}{N}+\sigma_{A S F}^{2}+\delta_{\text {temp }}^{2}+\sigma_{\text {temp }}^{2}
$$

When all the data arrays are available, algorithms within the software then test each grid point to see whether the eLoran signals meet certain acceptance criteria. With data at each point on the map, the integrity using HPL is calculated and plotted as shown in Fig. 8.

\section{CONCLUSION AND FUTURE WORK}

In this paper we presented a simulation-based proposal to establish the eLoran navigation system for the Egyptian marine sector. We have generated integrity plot for Egypt by installation of eLoran stations at candidate locations. The simulation results for this proposal of Egyptian eLoran achieved the IMO's integrity requirement over most of Egyptian"s coastal borders and harbors. The future work will be intended into studying the other factors affecting on the eLoran performance as a positioning system for Egyptian harbors including availability, continuity and accuracy. 


\section{REFERENCES}

[1] Grant A., Williams P., Ward N., and Basker S., GPS Jamming and the Impact on Maritime Navigation, The Journal of Navigation, 2009, Vol. 62, pp. 173-187.

[2] International Loran Association, Enhanced Loran (eLoran) Definition Document, 2007. v. 1.0.

[3] Jan Šafár: Analysis, Modelling and Mitigation of Cross-Rate Interference in Enhanced Loran, Ph.D thesis, Czech Technical University in Prague, August 2014.

[4] International Telecommunication Union, ITU-R Rec. 368-9, Ground- Wave Propagation Curves for Frequencies between $10 \mathrm{kHz}$ and $30 \mathrm{MHz}, 2007$.

[5] International Telecommunication Union, ITU-R P.372-9, Radio Noise, 2007.

[6] R. W. Nutting, Loran-C Rate Selection Process. United States Coast Guard, 1988.

[7] Brunavs P., Phase lags of $100 \mathrm{kHz}$ radio frequency ground wave and approximate formulas for computation, 1997.

[8] Millington, G, "Ground-wave Propagation over an Inhomogeneous Smooth Earth", Proceedings of the IEE, Series 96, Number 3, 1949.

[9] Last, J.D., Williams, P., Peterson, B. and Dykstra, K., „Propagation of Loran-C Signals in Irregular Terrain - Modelling and Measurements Part 1: Modellinge, 29th Annual Convention and Technical Symposium, International Loran Association, Washington DC, Washington USA, 13- 15 November 2000 .

Table 1: Primary Requirements for eLoran According to NPA and HPA

\begin{tabular}{|c|c|c|}
\hline Performance Requirement & NPA & HPA \\
\hline Accuracy & 307 meters, 2DRMS & 20 meters, 2 DRMS \\
\hline Monitor Limit/ Alert Limit & 556 meters & 50 meters, 2drms \\
\hline Integrity & $10^{-7} /$ hour & $3.10^{-5} /$ hour \\
\hline Time-to-Alert & 10 seconds & 10 seconds \\
\hline Availability & 99.9 to $99.99 \%$ & $99.7 \%$ \\
\hline Continuity & 99.9 to 99.99 & $99.85 \%$ over 3 hours \\
\hline
\end{tabular}

Table 2 : Egypt Loran Stations

\begin{tabular}{|l|c|c|c|}
\hline GRI & Station & Position & PWR(KW) \\
\hline \multirow{6}{*}{6731} & Menia & $27.5 \mathrm{~N}$ & 1000 \\
& (Master) & $30 \mathrm{E}$ & \\
\cline { 2 - 4 } & Matrouh & $31.5 \mathrm{~N}$ & 1000 \\
& (Secondary 1) & $26.2 \mathrm{E}$ & \\
\cline { 2 - 4 } & Port-Said & $30.92511 \mathrm{~N}$ & 1000 \\
& (Secondary 2) & $32.671567 \mathrm{E}$ & \\
\cline { 2 - 4 } & Ash-shaykh & $28.15444 \mathrm{~N}$ & 1000 \\
& (Secondary 3) & $34.76126 \mathrm{E}$ & \\
\cline { 2 - 4 } & Marsa-Alam & $24.88699 \mathrm{~N}$ & 1000 \\
& (Secondary 4) & $34.19199 \mathrm{E}$ & \\
\hline
\end{tabular}




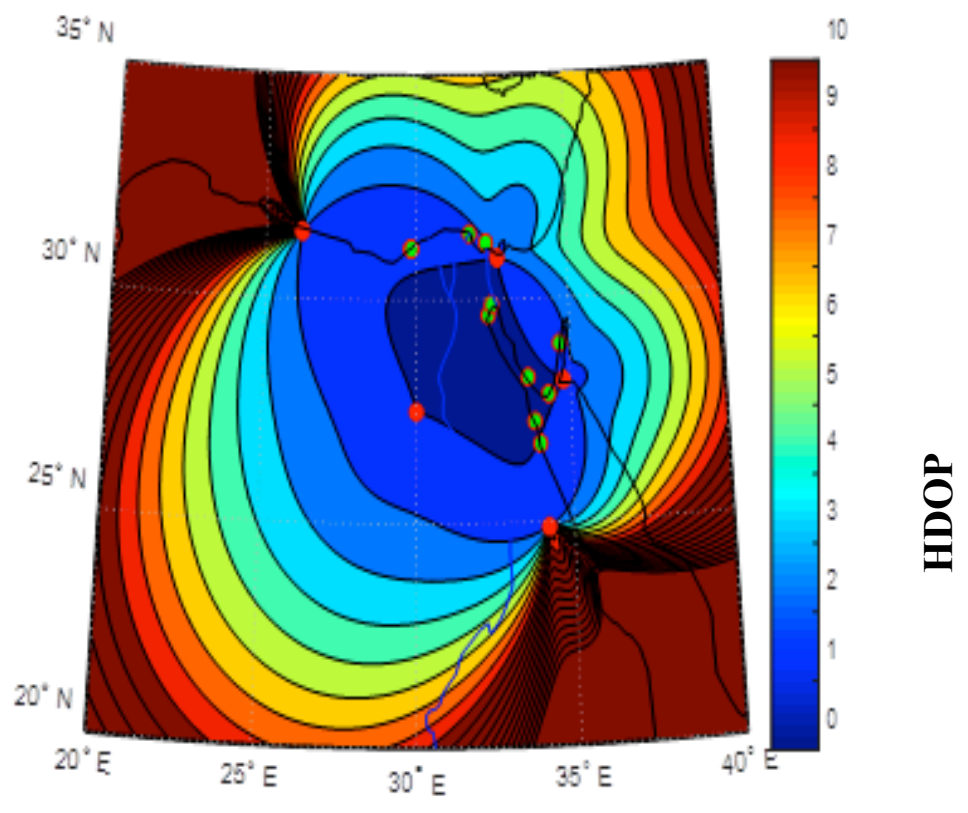

Fig. 1: HDOP distribution over Egypt using Loran transmitter stations, new transmitter are denoted by red points and harbors are denoted by yellow points

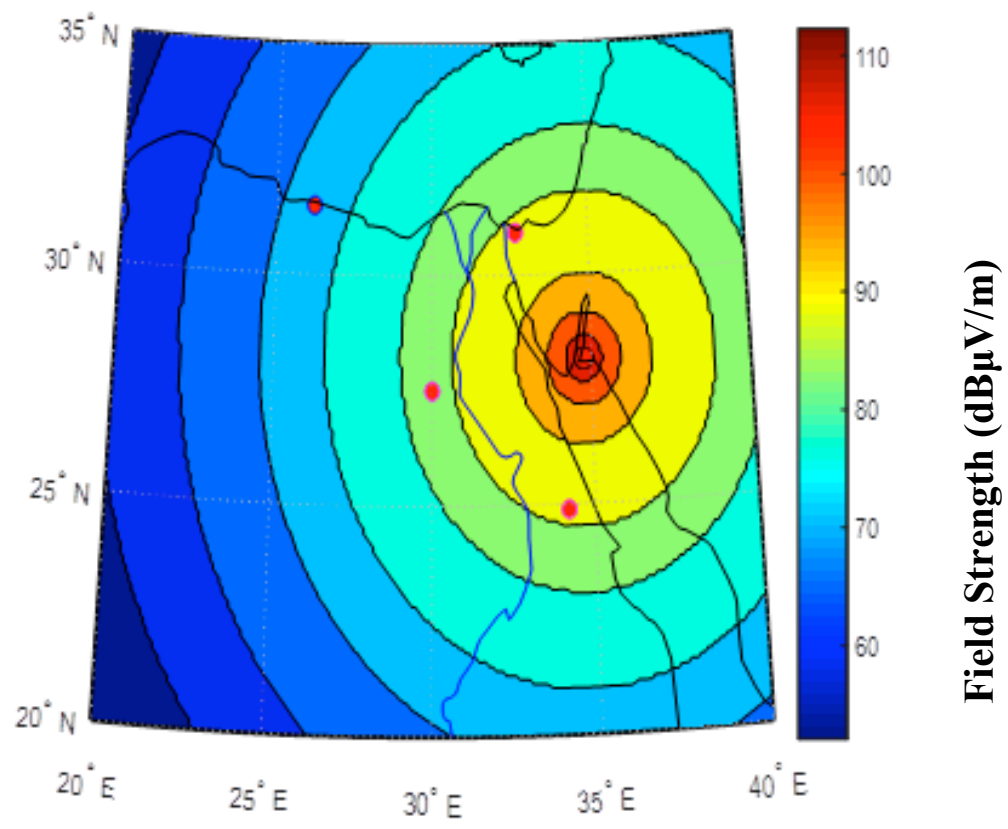

Fig. 2:Predicted ground wave field strength for the Ash-Shaykh station 


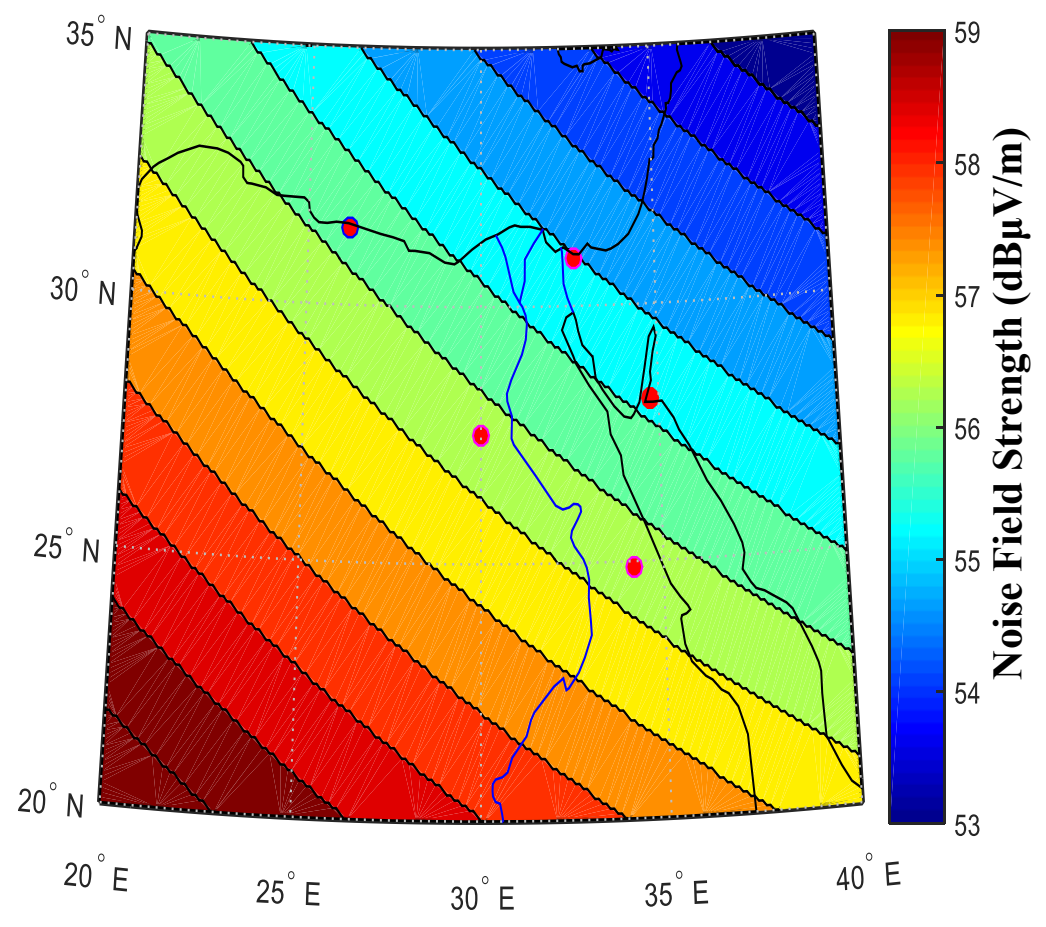

Fig. 3: Average daytime atmospheric noise over Egypt

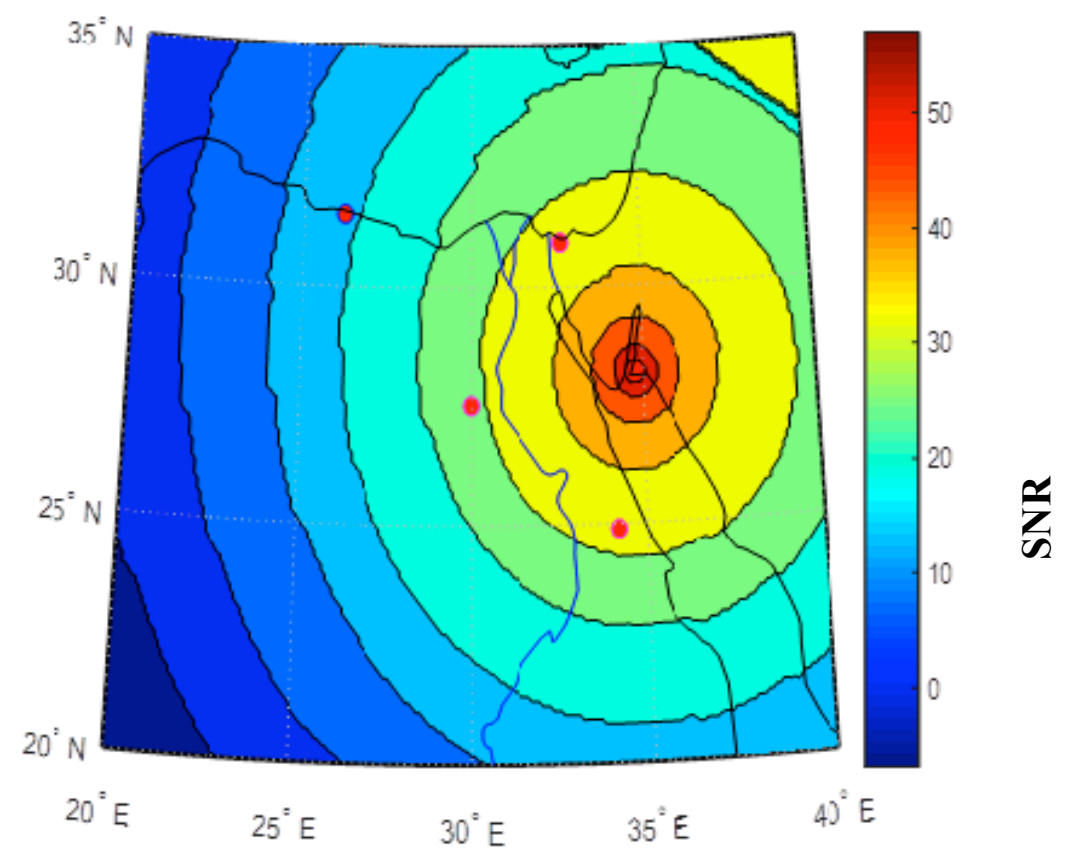

Fig. 4:Predicted SNR for the Ash-Shaykh eLoran station 


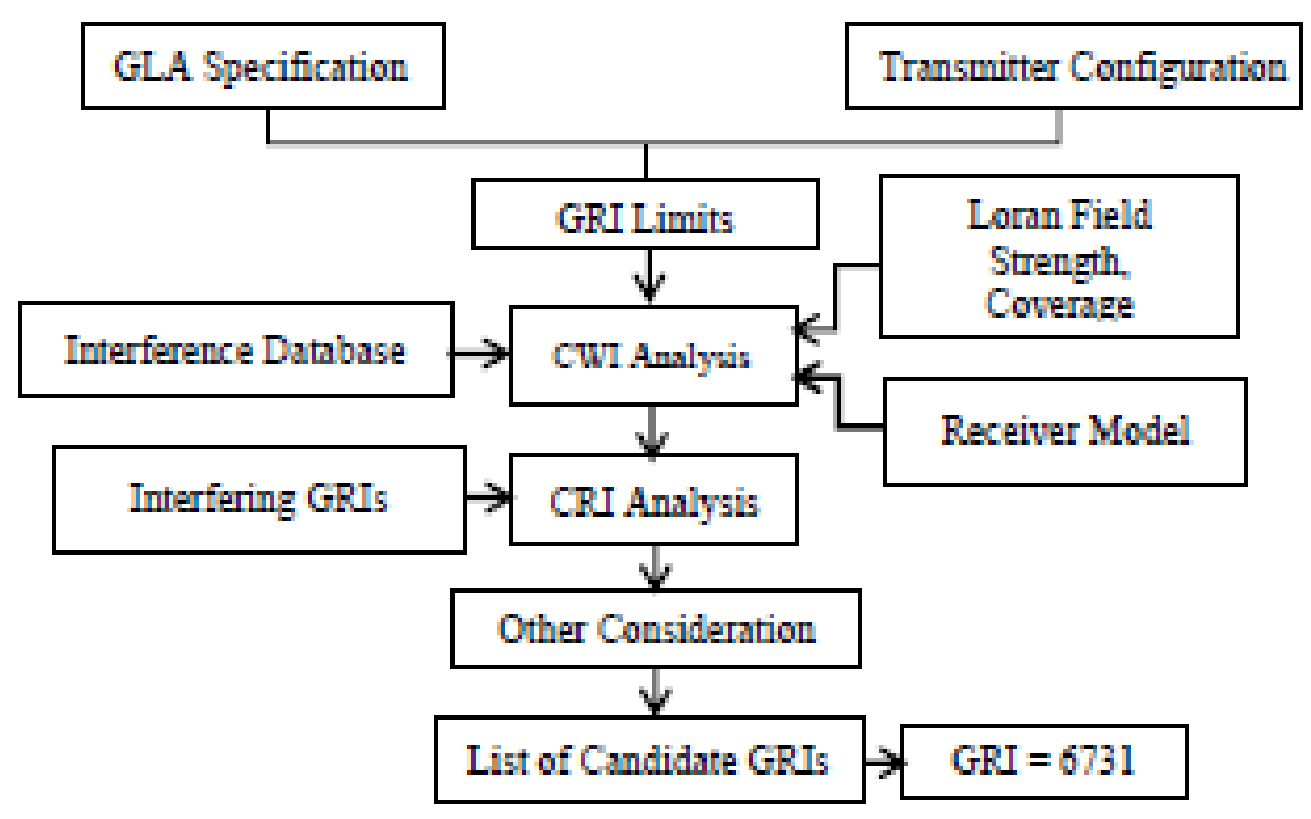

Fig .5: The overall procedure of GRI selection

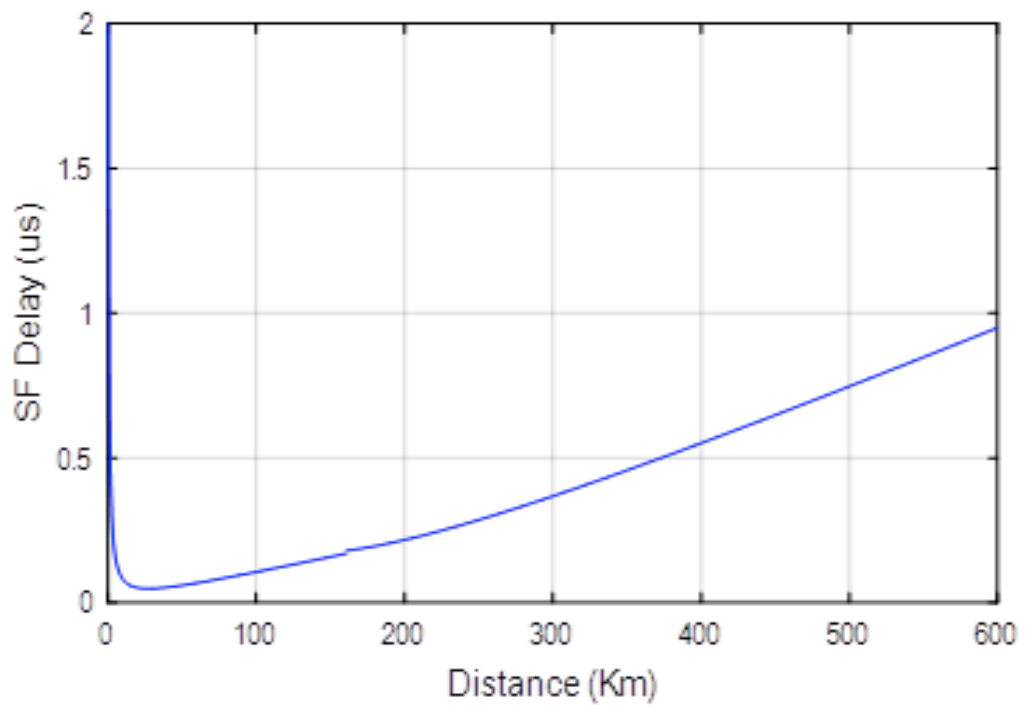

Fig. 6:Secondary Factor Delay from Brunavs' equations 


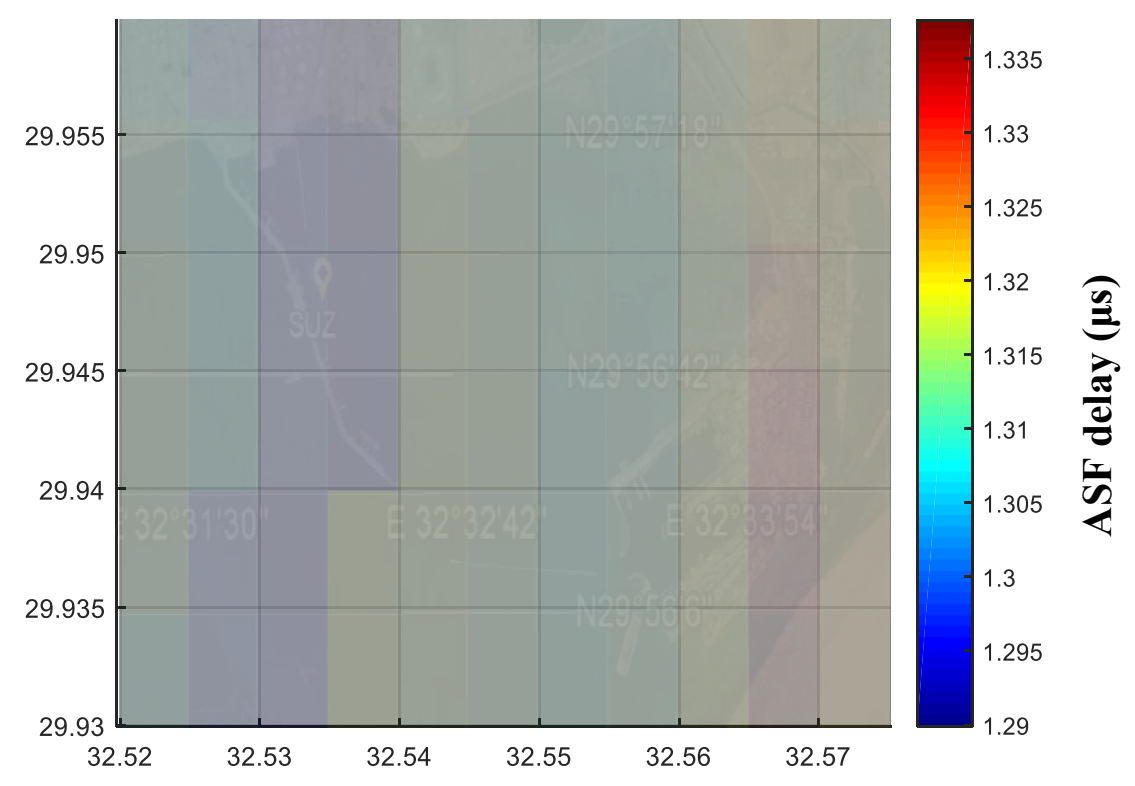

Fig. 7:Port Said Basic ASF grid of the data at 500m spacing measured at Suez port

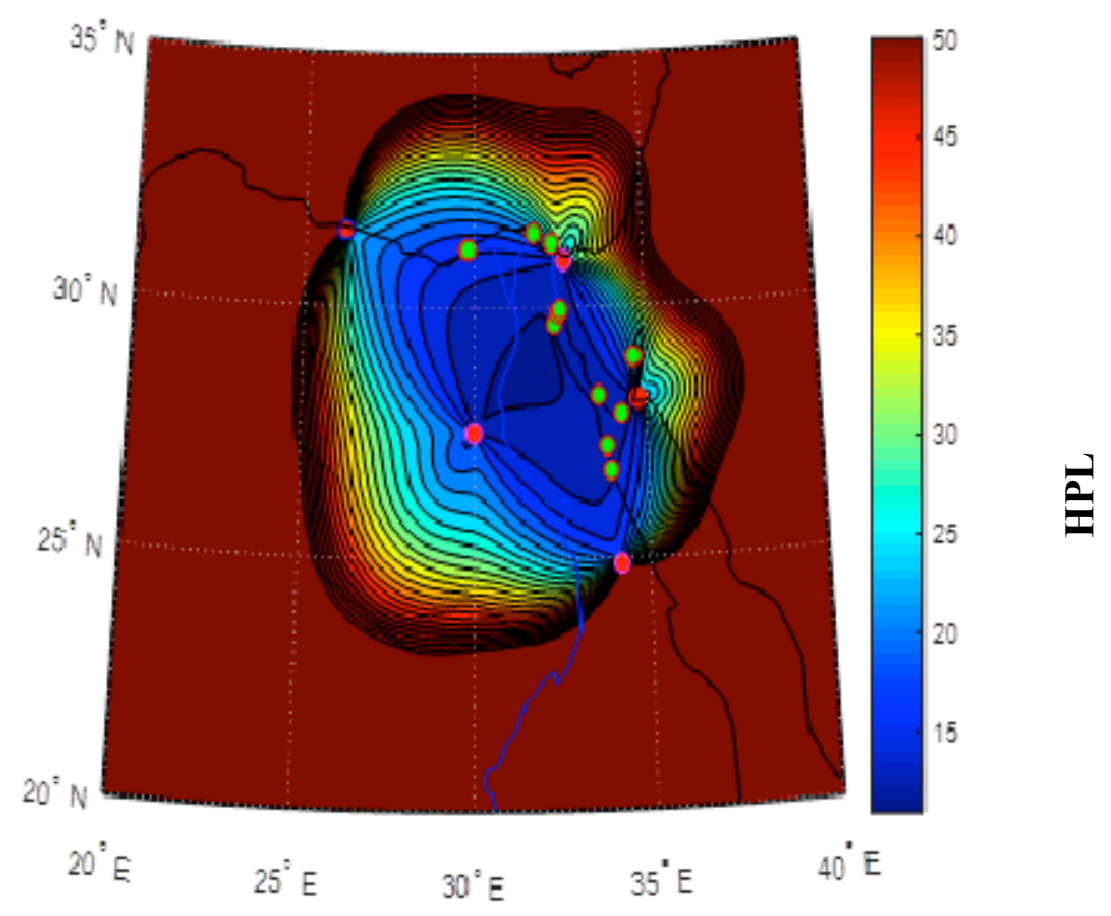

Fig. 8:Predicted Integrity of proposed eLoran chain 